\title{
Diversidad de la edafofauna de suelos cafeteros del sur de Colombia
}

\section{Diversity of the edaphic fauna of soils cultivated with coffee in southern Colombia}

\author{
Tulio Cesar Lagos-Burbano ${ }^{1^{\bullet}}$; William Ballesteros-Possu ${ }^{\circledR}$; Wilmer Libey Delgado-Gualmatan ${ }^{\circledR}$
}

Recibido para publicación: septiembre 24 de 2020 - Aceptado para publicación: diciembre 27 de 2020

\begin{abstract}
RESUMEN
La edafofauna es un indicador biológico de la fertilidad del suelo. Conocer su diversidad permite establecer relaciones funcionales con éste y tomar decisiones para establecer prácticas de conservación. El objetivo de este trabajo fue evaluar la riqueza (Ri), abundancia relativa (AR) y diversidad de la edafofauna en cuatro municipios del departamento de Nariño La Unión, Sandoná, Consaca y La Florida. Los muestreos se hicieron en tres altitudes que se tomaron como repeticiones. En bosques secundarios y cultivos de café se registraron la Ri y la AR en mantillo, entre 10 y 20 $\mathrm{cm}$ y entre 20 a $30 \mathrm{~cm}$ de profundidad. En Consacá se registraron un total de 19.836 individuos de 24 órdenes, de los cuales el $37,6 \%$ correspondieron a bosque y el $62,4 \%$ a café. La Florida mostró un total de 13.980 individuos de 16 órdenes de los cuales el $41,3 \%$ se ubicó en el bosque y el $58,7 \%$ en café. En La Unión se encontraron 6.935 individuos en 17 órdenes, donde el 64,2\% se ubicaron en el bosque y el $35,8 \%$ en café. El número de individuos en Sandoná fue de 10.436, de los cuales, el $10,7 \%$ se ubicaron en el bosque y el $89,3 \%$ en café. En los bosques se presentaron mayores valores de diversidad que en los cultivos café. La interacción sistemas $\mathrm{x}$ profundidades no fue significativa, a excepción de los índices de Sahnnon-Weaver y Simpson en Sandoná. Los órdenes Hymenoptera, Acari y Coleóptera fueron los más prevalentes con diferencias en Ri y AR entre bosque y café.
\end{abstract}

Palabras clave: Riqueza; Abundancia relativa; Bosque; Café; Shannon-Weaver; Simpson.

${ }^{1}$ Universidad de Nariño, Facultad de Ciencias Agrícolas, Grupo de Investigación en Producción de Frutales Andinos GPFA. San Juan de Pasto - Nariño, Colombia.

* Autor para correspondencia: Ph.D. Tulio Cesar Lagos-Burbano Email: tclagosb@udenar.edu.co

\begin{abstract}
The edaphic fauna is a biological indicator of soil fertility. Knowing its diversity allows establishing functional relationships with it and making decisions to establish conservation practices. The objective of this work was to evaluate the richness (Ri), relative abundance (AR), and diversity of the edaphic fauna in four municipalities of the department of Nariño, La Union, Sandona, Consaca y La Florida. The samplings were carried out at three altitudes, which were considered as replicates. In secondary forests and coffee crops, Ri and AR were recorded in mulch, between 10 and $20 \mathrm{~cm}$, and between 20 and $30 \mathrm{~cm}$ deep. In Consacá, were registered 19,836 individuals of 24 orders, of which $37.6 \%$ corresponded to forest and $62.4 \%$ to coffee. Florida showed 13,980 individuals from 16 orders of which $41.3 \%$ were located in the forest and $58.7 \%$ in coffee. In La Unión, were found 6,935 individuals in 17 orders, where $64.2 \%$ were located in the forest and $35.8 \%$ in coffee. The number of individuals in Sandoná was 10,436 , of which $10.7 \%$ were located in the forest and $89.3 \%$ in coffee. In forests, there were higher values of diversity than in coffee crops. The systems $\mathrm{x}$ depths interaction was not significant, an exception of the Shannon-Weaver and Simpson indices in Sandoná. Hymenoptera, Acari and Coleóptera were the most prevalent with differences in Ri and AR between forest and coffee.
\end{abstract}

Key words: Richness; Relative abundance; Forest; Coffee; Shannon-Weaver; Simpson.

\section{Cómo citar}

Lagos-Burbano, T.C., Ballesteros-Posuu, W. y Delgado-Bulmata, W. L. 2020. Diversidad de la edafofauna de suelos cafeteros del sur de Colombia. Temas Agrarios 25(2):117-128 https://doi.org/10.21897/rta.v25i2.2439

Temas Agrarios 2020. Este artículo se distribuye bajo los términos de la Licencia Creative Commons Attrubution 4.0 (https://creativecommons.org/licenses/ by-nc/4.0/deed.es), que permite copiar, redistribuir, remezclar, transformar y crear a partir del material, de forma no comercial, dando crédito y licencia de by-nc/4.0/deed.es), que permite copiar, redi 


\section{INTRODUCCIÓN}

El suelo es un recurso natural esencial que proporciona servicios eco sistémicos, en los cuales algunos invertebrados están involucrados (Lavelle, 2009). La fauna edáfica comprende a organismos con tamaños y estrategias adaptativas diferentes. Los de mayor tamaño, constituyen la macrofauna que contiene organismos con un ancho del cuerpo mayor a 2 $\mathrm{mm}$. La macrofauna se destaca porque directa o indirectamente afectan las propiedades del suelo. Las comunidades presentes son la consecuencia de las prácticas de manejo de suelo que se realizan, por lo que tienen gran potencial de uso como indicadores (Zerbino, 2010).

García y Bello (2004) indican que la biocenosis edáfica está constituida principalmente por la microflora (bacterias, algas y hongos), por la micro y mesofauna, y por algún grupo notable de macrofauna, como el de los lumbrícidos. La edafofauna comprende una gran variedad de organismos que intervienen en numerosos procesos del suelo, afectando tanto el crecimiento de la vegetación como el mantenimiento de la productividad (Masin et al., 2017).

La mesofauna y macrofauna desempeñan un papel clave en el funcionamiento del ecosistema, debido a que contribuyen con diferentes procesos del suelo (Aquino et al., 2012). Estos intervienen en los procesos de descomposición de la materia orgánica, de aceleración y reciclaje de nutrientes, en la mineralización los elementos escenciales (García y Bello, 2004) y en la regulación de las comunidades bióticas dentro o fuera del suelo (Brussaard, 2012); además, estos grupos son reguladores del proceso trófico del medio edáfico, al coadyuvar en la formación de su microestructura con sus aportes de deyecciones, excreciones, secreciones y con sus propios cadáveres (Socarrás, 2013).
La mesofauna y macrofauna constituyen factores decisivos para el mantenimiento de la productividad, aunque el uso de suelo asociado a la producción agrícola y ganadera es un factor clave en la biodiversidad edáfica (Cabrera-Mireles et al., 2019). En este sentido, Delgado et al., (2011) encontraron que la mayor abundancia y diversidad de macrofauna se presentó en los usos bosque, Coffea arabica intercalado con Musa sapientum, seguido del arreglo agroforestal $C$. arabica con Inga edulis y en menores porcentajes sistema $C$. arabica en monocultivo, el cual determinó los niveles más bajos.

La biodiversidad de dichos individuos declina en función inversa a la intensidad con que son cultivadas las plantas mediante métodos mecanizados y agroquímicos (García et al., 2014). La transformación de los ecosistemas naturales con fines agrícolas resulta en un impacto directo sobre las comunidades vegetales y la estructura física del suelo en donde provoca cambios negativos en la composición y la estructura de la fauna que lo habita, así como en el funcionamiento de este recurso y en general del ecosistema (Castellanos et al., 2019). Los distintos usos del suelo, así como el grado de perturbación e intensidad del manejo, influyen en la composición y la abundancia de la macrofauna edáfica. El uso intensivo del suelo en la explotación agrícola hace que disminuyan las comunidades de los organismos como consecuencia del efecto tóxico de los agroquímicos, la destrucción física de los hábitats y la reducción del alimento y de la materia orgánica (García et al., 2014).

Socarrás (2013) afirma que las poblaciones edáficas intervienen en la alteración de la materia orgánica, provocando una mayor velocidad en la mineralización, en especial del nitrógeno, fósforo y ciclaje de nutrientes; regulan procesos de conformación de las propiedades del suelo mediante sus aportes de excretas y secreciones, afectando además, la 
diseminación de otros microorganismos como hongos y bacterias.

Amazonas et al. (2018) indican que la macrofauna edáfica debe estudiarse mejor si se quiere aprovechar todo su potencial para la restauración de ecosistemas tropicales. Estos autores encontraron algunos patrones claros de cambio en la macrofauna del suelo, en consecuencia, la densidad no aumentó a lo largo de una sucesión secundaria, sino que se correlacionó con la cubierta del dosel. La diversidad se caracterizó por un alto dominio de los insectos sociales y la igualdad entre otros grupos; además, concluyen que la macrofauna tiene una alta capacidad para recolonizar los bosques jóvenes y su recuperación es considerablemente más rápida en comparación con otras transformaciones del ecosistema. Además de la importancia de conocer la macrofauna del suelo, el impacto del manejo de los agroecosistemas y los grupos de insectos indicadores de la calidad y fertilidad del suelo. (Santos et al., 2018).

Acorde con lo anterior, y dada la relevancia que tiene la edafofauna como indicador de fertilidad y calidad del suelo, el objetivo de este trabajo fue evaluar su riqueza, abundancia relativa y diversidad en dos usos de suelo, bosque y café.

\section{MATERIALES Y MÉTODOS}

La investigación se llevó a cabo en los ecotopos cafeteros 220A y 221A definidos como "espacios vitales en los que reinan condiciones ambientales similares" del departamento de Nariño. El municipio de La Unión del ecotopo 220A está ubicado en las coordenadas que van desde $1^{\circ} 21^{\prime}$ hasta $1^{\circ} 42^{\prime} \mathrm{LN}$, altitudes entre 1.300 y 1.800 msnm, con una precipitación anual de 1.700 a 1.900 mm. En el ecotopo 221A están los municipios de Sandoná, Consacá y La Florida, que hacen parte de la cuenca del río Guáitara, situados entre $1^{\circ} 05^{\prime}$ y $1^{\circ} 36^{\prime} \mathrm{LN}, 1.400$ y 2.100 msnm, con una precipitación anual de 1.400 a 1.700 mm. (Gómez et al., 1991).
Los muestreos se hicieron en los cuatro municipios antes mencionados en dos sistemas, uno de bosque secundario cercano al cultivo de café y otro de cultivo de café a libre exposición solar, variedad castillo de dos años aproximadamente de sembrado, de acuerdo con la metodología propuesta por Pardo-Locarno (2009) en tres sitios ubicados en tres altitudes, a $<1.500 \mathrm{msnm}$, entre 1.500 y $2000 \mathrm{msnm}$ y >2.000 msnm. En cada sitio de muestreo se abrieron tres monolitos de 0,25 x 0,25 x 0,20 m por cada uno de los sistemas (bosque y café). De cada monolito, se tomaron tres sub muestras, una superficial o en mantillo (M), la segunda 0 y $10 \mathrm{~cm}$ de profundidad (P1) y la tercera entre 10 y $20 \mathrm{~cm}$ de profundidad (P2). Cada sub muestra se colocó en una tela polietileno, para revisar y colectar en el campo todos los organismos visibles y colocarlos en alcohol del 70\%. Luego se guardaron en bolsas plásticas rotuladas, teniendo en cuenta la metodología Tropical Soil Biology and Fertility (Anderson e Ingram, 1994).

En el insectario de la Universidad de Nariño, para extraer la mesofauna del suelo, las muestras se dejaron en embudos de Berlese por un periodo de 96 horas. Los organismos se colectaron en un frasco que contenía mezcla de etanol, agua y glicerina en una proporción de 6:3:1. Los artrópodos se conservaron en alcohol al 70\% y los anélidos en formol al 5\%. Los organismos obtenidos fueron identificados por el laboratorio de entomología de la Universidad de Nariño hasta el nivel de orden.

Para cada uno de los dos sistemas evaluados, bosque y café, se obtuvo frecuencia de los individuos de cada taxón. Se determinó la riqueza, la abundancia relativa y se calcularon los índices de Shannon-Weaver y de Simpson, tal como se detalla a continuación:

Riqueza (Ri). Se expresó como el número de especies observadas en cada sistema. 
Abundancia absoluta (A). La $A$ determina el número de individuos por especie. Se obtuvo con la fórmula:

$$
\mathrm{A}=\frac{\mathrm{ni}}{\mathrm{N}}
$$

Dónde: $\mathrm{ni}$ = Número de individuos de la iésima especie, $\mathrm{N}=$ Número de individuos totales en la muestra.

Abundancia relativa (AR). Se calculó con base en la proporción de una especie o taxón respecto a todas las especies o taxones contenidos en un sitio, y se expresó en porcentaje. La AR se obtuvo de la siguiente manera:

$$
A R=\frac{\mathrm{n}_{i}}{\mathrm{~N}} \times 100
$$

Dónde: $\mathrm{n}_{\mathrm{i}}=$ número de individuos de la especie $i, \mathrm{~N}=$ total de individuos de todas las especies.

Índice de Shannon-Weaver $(\boldsymbol{H})$. Mide la diversidad específica, toma valores que varían entre 1 y 4.5, valores por encima de 3 son considerados como diversos, acorde con la siguiente ecuación:

$$
\mathrm{H}=-\sum_{i=1}^{S} \mathrm{P}_{i} x \ln \mathrm{P}_{i}
$$

Dónde: $\mathrm{Pi}=$ proporción de individuos de la especie $i$ respecto al total de individuos, que corresponde a la abundancia relativa de la especie $i, \mathrm{~s}=$ número de especies o riqueza de las especies (Jost, 2006).

Índice de diversidad de Simpson (Ds). Es el índice de la diversidad de las especies o índice de dominancia. Mide la riqueza de los organismos y se calcula de la siguiente forma:

$$
D s=\frac{\sum_{i=1}^{S} n_{i}\left(n_{i}-1\right)}{N(N-1)}
$$

Dónde: $\mathrm{s}=$ número de especies, $\mathrm{N}=$ total de organismos presentes, $\mathrm{ni}=$ número total de individuos de la especie $i$ (Jost, 2006).
En cuanto a la riqueza, en cada ítem, se dio una calificación de uno a cinco. Uno para el menor valor, dos al segundo de menor valor, y así sucesivamente hasta cinco, que corresponde al máximo valor. Luego se sumaron las calificaciones para una calificación total. El municipio y la profundidad con la mayor calificación total se consideraron como los de mayor riqueza. Para cada municipio se hizo el Análisis de Varianza (ANDEVA) bajo el modelo del diseño de Bloque Completos al azar con dos factores (Steel y Torrie, 1960) y tres repeticiones, las cuales correspondieron a las altitudes. En el factor uno se consideraron dos sistemas, uno de bosque secundario y otro de cultivo de café y en el factor dos las profundidades (mantillo (M), P1 y P2), se empleó la prueba no paramétrica de Wilcoxon, la cual permite comparar el rango medio de dos muestras relacionadas y determinar si existen diferencias entre ellas.

\section{RESULTADOS Y DISCUSIÓN}

En la localidad de Consacá se observaron diferencias significativas entre los sistemas en Riqueza (Ri), abundancia absoluta (A) y abundancia relativa (AR). En profundidad, existieron diferencias en $\mathrm{Ri}, \mathrm{A}$ y $\mathrm{AR}$ (Tabla 1). La abundancia absoluta fue altamente correlacionada con la $A R$, dado que ésta se generó a partir de la primera, por lo tanto, en ésta localidad y para las demás, sólo se discutirá la AR. Tanto la Ri como la AR, estadísticamente, fueron mayores en el bosque que en el cultivo de café. El M superó a P1 y P2, así mismo, P1 fue estadísticamente mayor a $\mathrm{P} 2$, mientras que en AR fueron similares (Tabla 2).

En La Florida sólo existieron diferencias en la Ri (Tabla 1), donde $M$, al igual que Consacá, presentó los mayores promedios en comparación con el cultivo de café. M y P1 fueron estadísticamente iguales pero con diferencias al compararlo con P2 que fue el de menor promedio. Los promedios de AR fueron iguales estadísticamente con un promedio 
de $5,60 \%$ y valores que oscilaron entre 3,40 y $9,80 \%$, lo cual significa que no existieron órdenes dominantes o muy frecuentes (Tabla 2).

En La Unión, solamente se encontraron diferencias en los niveles de profundidad de la Ri. En las demás variables no existieron diferencias. El promedio de Ri fue mayor en $\mathrm{M}$ que en $\mathrm{P} 1$ y $\mathrm{P} 2$, mientras que entre estas últimas no se observaron diferencias. La AR fue de 5,6\% con un rango entre 3,8 y 5,70\%. En Sandoná no se obtuvieron diferencias significativas en la Ri y en la AR. Los valores de Ri oscilaron entre 7,7 y 10,7 órdenes y los de la AR entre 3,2 y 5,6\%. Este comportamiento es similar a las anteriores localidades (Tablas 1 y 2 ).

En términos generales, los valores de $\mathrm{Ri} y$ AR fueron semejantes en las localidades, profundidades, sistemas de bosque y café con pocas diferencias estadísticas significativas. En estos espacios, el número de órdenes osciló entre 7 y 16,3. Estos valores son similares a los obtenidos en otros estudios. Al respecto, se ha encontrado que la riqueza taxonómica a nivel de familias, presentó el mismo número en un bosque secundario y en un sistema silvopastoril (13); mientras que en un pastizal cultivado se reportaron nueve familias (Socarrás-Rivero, 2018). En plantaciones de arándanos (Vaccinium sp.) sometidos a manejo orgánico y convencional en fincas del centrosur de Chile, se reportó que la riqueza de los taxones fue similar en todas las plantaciones, independientemente del manejo agrícola (Peredo et al., 2009), lo cual es semejante a lo reportado en este estudio.

La interacción sistema $x$ profundidad (ISP) no fue significativa para Ri y AR en todos los municipios (Tabla 1). Resultados semejantes encontraron Gizzi et al. (2009), quienes determinaron que la fauna edáfica no mostró interacción significativa entre sistemas de cultivo; sin embargo, los sistemas de cultivo si afectaron la composición taxonómica y la abundancia relativa de la meso y macrofauna del suelo. En suelos bajo agricultura, los indicadores de la fauna edáfica disminuyen con distinta intensidad según el sistema de labranza (Gizzi et al., 2009). Los grupos de la mesofauna del suelo son muy sensibles a cambios que ocurren en el medio edáfico por causas naturales o antrópicas, lo que provoca variaciones en su densidad y diversidad (Socarrás, 2013). Lo mismo puede darse en los sistemas de cultivo de café y de bosque de las zonas cafeteras del departamento de Nariño, dadas las diferencias en la forma de cultivar el café y en la influencia antrópica en los bosques secundarios.

Acorde con lo anterior Gómez et al. (1991) identificaron dos ecotopos cafeteros para el departamento de Nariño, el 220A y 221A. Al 220A pertenece el municipio de la Unión, donde los suelos son superficiales con bajo contenido de materia orgánica, fertilidad natural media y alta susceptibilidad a la erosión. La caficultura se hace con sombrío muy disperso en sectores con mayor concentración de café alrededor de la cabecera municipal de La Unión, bajo la presencia de cenizas volcánicas. Al ecotopo 221A corresponden Consacá, La Florida y Sandoná, cuyos suelos tienen baja capacidad de retención de humedad y alta susceptibilidad a la erosión. La mayor parte de la caficultura se hace con sombrío e intercalados con plátano y menor proporción de sectores con cenizas volcánicas.

En Consacá se registraron un total de 19.836 individuos de 24 órdenes, de los cuales el $37,6 \%$ correspondieron a bosque y el $62,4 \%$ a café. Los órdenes de mayor AR fueron Hymenoptera (40,81\%), Acari $(20,7 \%)$, Isopoda $(15,3 \%)$, Coleoptera (5,6\%), Haplotaxida $(5,5 \%)$ y Polydesmida (3,3\%), los cuales representan el $91,2 \%$ del total de individuos. En el sistema bosque, los más abundantes fueron Hymenoptera con $26,4 \%$ en $M$ y $30 \%$ en P2 y Acari en P1 (17,9\%). En el sistema café, también el orden Hymenoptera fue el de mayor AR en todas las profundidades con el $57,8 \%$ en M, 32,2\% en P1 y 59,2\% den P2 (Tabla 3). 
La Florida mostró un total de 13.980 individuos distribuidos en 16 órdenes. El 41,3\% se ubicó en el bosque y el 58,7\% en café. Los órdenes más abundantes fueron Hymenoptera (51,4\%), Acari (7,6\%), Coleóptera (18,3\%), Haplotaxida $(6,9 \%)$, Polydesmida $(4,7 \%)$, y Hemiptera (2,3\%). Estos órdenes contienen el 91,82\% del total de individuos. En el estrato $M$ de bosque los taxones de mayor AR fueron Acari con
29,5\% e Hymenoptera con 23,9\%. Para P1, los taxones de mayor AR fueron Coleoptera $(33,3 \%)$ e Hymenoptera (21,3\%). Similar comportamiento tuvieron los mismos taxones en la profundidad P2, donde presentaron porcentajes de 31,1 y $44 \%$, respectivamente. En el sistema de producción café los taxones de mayor $A R$ en $M$ fueron Hymenoptera con $38,9 \%$ y Acari con $20,8 \%$ (Tabla 3 ).

Tabla 1. Cuadrados medios del Análisis de varianza (ANDEVA) para la riqueza (Ri), abundancia absoluta (A, abundancia relativa (AR), índice de Shannon-Weaver $(\mathrm{H})$ e índice de Simpson (Ds) de la edafofauna de cuatro municipios cafeteros del departamento de Nariño.

\begin{tabular}{|c|c|c|c|c|c|c|}
\hline \multicolumn{7}{|l|}{ Consacá } \\
\hline FV & $G L$ & $R i$ & $A$ & $A R$ & $H$ & Ds \\
\hline Repetición & 2 & 1,06 & 577004,17 & 9,36 & 2,45 & 0,11 \\
\hline Sistema (S) & 1 & $64,22 * *$ & $2070612,50^{* *}$ & $33,60^{* *}$ & $0,11 * *$ & $0,012 * *$ \\
\hline Profundidad (P) & 2 & $102,39 * *$ & $5072929,17 * *$ & $82,32 * *$ & 0,007 & 0,02 \\
\hline $\mathrm{SxP}$ & 2 & 12,06 & 400879,17 & 6,50 & 2,45 & 0,11 \\
\hline Error & 10 & 3,26 & 204662,50 & 3,32 & 0,07 & 0,011 \\
\hline $\mathrm{CV}(\%)$ & & 15,18 & 32,80 & 32,78 & 17,61 & 32,65 \\
\hline Media & & 11,90 & 1379,17 & 5,56 & 1,48 & 0,31 \\
\hline \multicolumn{7}{|l|}{ La Florida } \\
\hline$F V$ & $G L$ & $R i$ & $A$ & $A R$ & $\boldsymbol{H}$ & Ds \\
\hline Repetición & 2 & 2,39 & 326679,17 & 10,62 & 0,10 & 0,002 \\
\hline Sistema (S) & 1 & $76,06^{* *}$ & 498334,72 & 16,19 & $2,17^{* *}$ & 0,12 \\
\hline Profundidad (P) & 2 & $30,89 * *$ & 620579,17 & 20,19 & $1,03 * *$ & 0,061 \\
\hline SxP & 2 & 4,22 & 1184184,72 & 38,53 & 0,19 & 0,06 \\
\hline Error & 10 & 2,39 & 743994,17 & 24,19 & 0,09 & 0,06 \\
\hline CV $(\%)$ & & 12,04 & 88,54 & 88,53 & 22,92 & 62,25 \\
\hline Media & & 9,06 & 974,17 & 5,56 & 1,78 & 0,39 \\
\hline \multicolumn{7}{|l|}{ La Unión } \\
\hline$F V$ & $G L$ & $R i$ & $A$ & $A R$ & $\boldsymbol{H}$ & Ds \\
\hline Repetición & 2 & 1,39 & 371984,72 & 31,50 & 0,62 & 0,14 \\
\hline Sistema (S) & 1 & 9,39 & 4512,50 & 0,38 & 0,11 & 0,10 \\
\hline Profundidad (P) & 2 & $33,39^{*}$ & 392293,06 & 33,21 & 0,60 & 0,07 \\
\hline SxP & 2 & 1,06 & 765129,17 & 64,79 & 0,06 & 0,04 \\
\hline Error & 10 & 2,79 & 371984,72 & 50,29 & 0,20 & 0,16 \\
\hline CV $(\%)$ & & 21,02 & 127,31 & 127,64 & 35,38 & 95,34 \\
\hline Media & & 7,94 & 603,61 & 5,56 & 1,26 & 0,42 \\
\hline \multicolumn{7}{|l|}{ Sandoná } \\
\hline$F V$ & $G L$ & $R i$ & $A$ & $A R$ & $\boldsymbol{H}$ & Ds \\
\hline Repetición & 2 & 4,50 & 96130,50 & 2,29 & 0,19 & 0,046 \\
\hline Sistema (S) & 1 & 24,50 & 420,50 & 0,009 & $0,42 * *$ & 0,025 \\
\hline Profundidad (P) & 2 & 17,17 & 1024722,17 & 24,39 & $0,50 * *$ & $0,061^{*}$ \\
\hline $\mathrm{SxP}$ & 2 & 3,50 & 1115817,17 & 26,57 & $0,54 * *$ & $0,085^{*}$ \\
\hline Error & 10 & 5,37 & 653044,83 & 15,54 & 0,053 & 0,015 \\
\hline CV $(\%)$ & & 24,39 & 70,98 & 70,96 & 17,76 & 29,82 \\
\hline Media & & 9,5 & 1138,50 & 5,56 & 1,30 & 0,42 \\
\hline
\end{tabular}

* Significativo, ** altamente significativo $\mathbf{C V}=$ coeficiente de variación, Sistema = bosque y café. 
Tabla 2. Prueba de comparación de medias de Wilcoxon, para riqueza (Ri), abundancia absoluta(A), abundancia relativa (AR), índice de Shannon-Weaver $(\mathrm{H})$ e índice de Simpson (Ds) de la edafofauna de cuatro municipios cafeteros del departamento de Nariño.

\begin{tabular}{lccccc}
\hline Consacá & $\boldsymbol{R} \boldsymbol{i}$ & $\boldsymbol{A}$ & $\boldsymbol{A R}$ & $\boldsymbol{H}$ & $\boldsymbol{D} \boldsymbol{s}$ \\
\hline Bosque & $13,78 \mathrm{a}$ & $1718,3 \mathrm{a}$ & $6,92 \mathrm{a}$ & $1,85 \mathrm{a}$ & $0,39 \mathrm{a}$ \\
Café & $10,00 \mathrm{~b}$ & $1040,0 \mathrm{~b}$ & $4,19 \mathrm{~b}$ & $1,11 \mathrm{~b}$ & $0,24 \mathrm{~b}$ \\
M & $16,33 \mathrm{a}$ & $2440,8 \mathrm{a}$ & $9,83 \mathrm{a}$ & $1,56 \mathrm{a}$ & $0,36 \mathrm{a}$ \\
P1 & $11,17 \mathrm{~b}$ & $860,0 \mathrm{~b}$ & $3,46 \mathrm{~b}$ & $1,56 \mathrm{a}$ & $0,31 \mathrm{a}$ \\
P2 & $8,17 \mathrm{c}$ & $836,7 \mathrm{~b}$ & $3,37 \mathrm{~b}$ & $1,32 \mathrm{a}$ & $0,27 \mathrm{a}$ \\
\hline $\boldsymbol{L a}$ Florida & $\boldsymbol{R i}$ & $\boldsymbol{A}$ & $\boldsymbol{A R}$ & $\boldsymbol{H}$ & $\boldsymbol{D} \boldsymbol{s}$ \\
\hline Bosque & $11,11 \mathrm{a}$ & $1140,6 \mathrm{a}$ & $6,50 \mathrm{a}$ & $1,63 \mathrm{a}$ & $0,47 \mathrm{a}$ \\
Café & $7,00 \mathrm{~b}$ & $807,8 \mathrm{a}$ & $4,61 \mathrm{a}$ & $0,93 \mathrm{~b}$ & $0,31 \mathrm{a}$ \\
M & $10,83 \mathrm{a}$ & $1345,0 \mathrm{a}$ & $7,67 \mathrm{a}$ & $1,63 \mathrm{a}$ & $0,49 \mathrm{a}$ \\
P1 & $9,83 \mathrm{a}$ & $805,8 \mathrm{a}$ & $4,60 \mathrm{a}$ & $1,38 \mathrm{a}$ & $0,40 \mathrm{a}$ \\
P2 & $6,60 \mathrm{~b}$ & $771,7 \mathrm{a}$ & $4,40 \mathrm{a}$ & $0,82 \mathrm{~b}$ & $0,29 \mathrm{a}$ \\
\hline $\boldsymbol{L a}$ Unión & $\boldsymbol{R i}$ & $\boldsymbol{A}$ & $\boldsymbol{A R}$ & $\boldsymbol{H}$ & $\boldsymbol{D} \boldsymbol{S}$ \\
\hline Bosque & $8,67 \mathrm{a}$ & $619,4 \mathrm{a}$ & $5,70 \mathrm{a}$ & $1,34 \mathrm{a}$ & $0,50 \mathrm{a}$ \\
Café & $7,22 \mathrm{a}$ & $587,8 \mathrm{a}$ & $5,41 \mathrm{a}$ & $1,18 \mathrm{a}$ & $0,35 \mathrm{a}$ \\
M & $10,67 \mathrm{a}$ & 895,0 & $8,24 \mathrm{a}$ & $1,60 \mathrm{a}$ & $0,53 \mathrm{a}$ \\
P1 & $6,67 \mathrm{~b}$ & 499,2 & $4,60 \mathrm{a}$ & $1,22 \mathrm{a}$ & $0,43 \mathrm{a}$ \\
P2 & $6,50 \mathrm{~b}$ & 416,7 & $3,84 \mathrm{a}$ & $0,97 \mathrm{a}$ & $0,31 \mathrm{a}$ \\
\hline Sandoná & $\boldsymbol{R i}$ & $\boldsymbol{A}$ & $\boldsymbol{A R}$ & $\boldsymbol{H}$ & $\boldsymbol{D S}$ \\
\hline Bosque & $10,67 \mathrm{a}$ & $1143,3 \mathrm{a}$ & $5,58 \mathrm{a}$ & $1,45 \mathrm{a}$ & $0,45 \mathrm{a}$ \\
Café & $8,33 \mathrm{a}$ & $1133 \mathrm{a}$ & $5,53 \mathrm{a}$ & $1,14 \mathrm{a}$ & $0,38 \mathrm{a}$ \\
M & $11,00 \mathrm{a}$ & $1381,7 \mathrm{a}$ & $6,74 \mathrm{a}$ & $1,50 \mathrm{a}$ & $0,53 \mathrm{a}$ \\
P1 & $9,83 \mathrm{ab}$ & $1372,5 \mathrm{a}$ & $6,70 \mathrm{a}$ & $1,43 \mathrm{a}$ & $0,36 \mathrm{~b}$ \\
P2 & $7,67 \mathrm{~b}$ & $661,3 \mathrm{a}$ & $3,23 \mathrm{a}$ & $0,96 \mathrm{~b}$ & $0,35 \mathrm{~b}$ \\
\hline & & & &
\end{tabular}

Medias iguales no son diferentes estadísticamente, $\mathrm{M}=$ mantillo, $\mathrm{P} 1=$ profundidad entre 0 y $10 \mathrm{~cm}, \mathrm{P} 2=$ profundidad entre 10 y $20 \mathrm{~cm}$.

En La Unión se encontraron 6.935 individuos en 17 órdenes. Al contrario que en Consacá y La Florida, en ésta localidad el 64,2\% de los individuos se encontraron en el bosque y el 35,8\% en café. Además, los órdenes más abundantes fueron Hymenoptera (65\%), Coleoptera $(9,11 \%)$, Haplotaxida $(6,6 \%)$, Hemiptera $(5,8 \%)$ y Polydesmida $(3,9 \%)$, los cuales suman el 90,4\%. El orden Hymenoptera es el de mayor AR del sistema bosque con el $61,9 \%$ en $\mathrm{M}$, el $59,9 \%$ en $\mathrm{P} 1$ y el $88,9 \%$ en $\mathrm{P} 2$. En café, también el orden Hymenoptera fue el de mayor AR con el 34,3\% en $M$, el $67,2 \%$ en P1 y el 48,7 en P2 (Tabla 3).

El número de individuos que se encontraron en Sandoná fue de 10.436, de los cuales, el
$10,7 \%$ se ubicaron en el bosque y el $89,3 \%$ en café, valores que son disimiles a La Unión y con la misma tendencia de Consacá y La Florida, pero con mayores diferencias en la proporción. Se encontraron 16 órdenes, donde Hymentoptera $(36,3 \%)$, Acari $(36 \%)$, Díptera $(8,3 \%)$, Haplotaxida (8,2\%) Coleóptera (3,7\%), fueron los de mayor AR con el 92,48\% de los individuos observados. Los órdenes que muestran mayor proporción para el sistema bosque se encuentran Acari con un 27,5\% en M. En P1 y P2, el orden de mayor presencia fue Hymenoptera con 56,6\%. En café, el orden de mayor proporción en $\mathrm{M}$ fue Acari con una $\mathrm{AR}$ del 55,4\%, para P1 y P2, Hymenoptera fue el orden de mayor AR con 31,6 y $62,8 \%$, en su orden (Tabla 3). 
Tabla 3. Ordenes de la edafofauna con mayor abundancia relativa en cuatro municipios cafeteros del departamento de Nariñocafeteros del departamento de Nariño.

\begin{tabular}{|c|c|c|c|c|c|c|c|c|c|c|c|c|}
\hline \multirow[b]{3}{*}{ Orden } & \multicolumn{6}{|c|}{ Bosque } & \multicolumn{6}{|c|}{ Café } \\
\hline & \multicolumn{2}{|c|}{$\bar{M}$} & \multicolumn{2}{|c|}{ P1 } & \multicolumn{2}{|c|}{$\mathbf{P 2}$} & \multicolumn{2}{|c|}{$\mathbf{M}$} & \multicolumn{2}{|c|}{ P1 } & \multicolumn{2}{|c|}{$\mathbf{P 2}$} \\
\hline & $\mathbf{N}$ & $\mathbf{A R}$ & $\mathbf{N}$ & $\mathbf{A R}$ & $\mathbf{N}$ & $\mathbf{A R}$ & $\mathbf{N}$ & $\mathbf{A R}$ & $\mathbf{N}$ & AR & $\mathbf{N}$ & AR \\
\hline \multicolumn{13}{|l|}{ Consacá } \\
\hline Hymenoptera & 1156 & 26,4 & 412 & 23,0 & 396,0 & 30,4 & 4240,0 & 57,8 & 1300,0 & 32,2 & 592,0 & 59,2 \\
\hline Acari & 1096 & 25,1 & 320 & 17,9 & 44,0 & 3,4 & 1920,0 & 26,2 & 588,0 & 14,6 & 136,0 & 13,6 \\
\hline Isopoda & 580 & 13,3 & 40 & 2,24 & 392,0 & 30,1 & 312,0 & 4,3 & 1704,0 & 42,3 & 0,0 & 0,0 \\
\hline Coleóptera & 444 & 10,2 & 216 & 12,1 & 96,0 & 7,4 & 188,0 & 2,6 & 80,0 & 1,98 & 88,0 & 8,8 \\
\hline Polydesmida & 308 & 7,0 & 164 & 9,17 & 60,0 & 4,6 & 92,0 & 1,3 & 20,0 & 0,5 & 4,0 & 0,4 \\
\hline Haplotaxida & 72 & 1,7 & 256 & 14,30 & 164,0 & 12,6 & 232,0 & 3,2 & 264,0 & 6,6 & 112,0 & 11,2 \\
\hline Total & 4372 & 100 & 1788 & 100,0 & 1304,0 & 100,0 & 7340,0 & 100,0 & 4032,0 & 100,0 & 1000,0 & 100,0 \\
\hline
\end{tabular}

La Florida

\begin{tabular}{|c|c|c|c|c|c|c|c|c|c|c|c|c|}
\hline Acari & 776 & 29,5 & 16 & 0,8 & 4,0 & 0,3 & 220,0 & 20,8 & 20,0 & 0,4 & 28,0 & 1,1 \\
\hline Hymenoptera & 628 & 23,9 & 412 & 21,3 & 532,0 & 44,0 & 412,0 & 38,9 & 3792,0 & 84,4 & 1404,0 & 52,8 \\
\hline Polydesmida & 268 & 10,2 & 288 & 14,9 & 64,0 & 5,3 & 8,0 & 0,8 & 12,0 & 0,3 & 16,0 & 0,6 \\
\hline Coleóptera & 244 & 9,3 & 644 & 33,3 & 376,0 & 31,1 & 104,0 & 9,8 & 316,0 & 7,0 & 872,0 & 32,8 \\
\hline Haplotaxida & 140 & 5,3 & 132 & 6,8 & 116,0 & 9,6 & 112,0 & 10,6 & 168,0 & 3,7 & 300,0 & 11,3 \\
\hline Hemiptera & 92 & 3,5 & 44 & 2,3 & 8,0 & 0,7 & 140,0 & 13,2 & 104,0 & 2,3 & 24,0 & 0,9 \\
\hline Total & 2628 & 100 & 1932 & 100,0 & 1208,0 & 100,0 & 1060,0 & 100,0 & 4492,0 & 100,0 & 2660,0 & 100,0 \\
\hline \multicolumn{13}{|l|}{ La Unión } \\
\hline Hymenoptera & 820 & 61,9 & 628 & 59,9 & 1852,0 & 88,9 & 420,0 & 34,3 & 640,0 & 67,2 & 148,0 & 48,7 \\
\hline Coleóptera & 116 & 8,8 & 96 & 9,2 & 92,0 & 4,4 & 200,0 & 16,3 & 60,0 & 6,3 & 68,0 & 22,4 \\
\hline Poly & 80 & 6,0 & 56 & 5,3 & 20,0 & 1,0 & 48,0 & 3,92 & 52,0 & 5,5 & 12,0 & 4,0 \\
\hline Haplotaxida & 56 & 4,2 & 160 & 15,3 & 68,0 & 3,3 & 84,0 & 6,86 & 60,0 & 6,3 & 32,0 & 10,5 \\
\hline Hemiptera & 24 & 1,8 & 24 & 2,3 & 8,0 & 0,4 & 256,0 & 20,9 & 76,0 & 7,98 & 16,0 & 5,3 \\
\hline Total & 1324 & 100 & 1048 & 100,0 & 2083,0 & 100,0 & 1224,0 & 100,0 & 952,0 & 100,0 & 304,0 & 100,0 \\
\hline \multicolumn{13}{|l|}{ Sandoná } \\
\hline Acari & 112 & 21,0 & 320 & 64,5 & 28,0 & 13,2 & 2740,0 & 55,4 & 232,0 & 13,4 & 320,0 & 12,1 \\
\hline Coleóp & 60 & 14,7 & 16 & 3,2 & 16,0 & 7,6 & 160,0 & 3,24 & 80,0 & 4,62 & 56,0 & 2,1 \\
\hline Hymenoptera & 56 & 13,7 & 32 & 6,5 & 120,0 & 56,6 & 1372,0 & 27,8 & 548,0 & 31,6 & 1660,0 & 62,8 \\
\hline Díptera & 24 & 5,9 & 12 & 2,4 & 0,0 & 0,0 & 192,0 & 3,88 & 360,0 & 20,8 & 276,0 & 10,4 \\
\hline Haplotax & 4 & 1,0 & 12 & 2,4 & 16,0 & 7,6 & 192,0 & 3,88 & 360,0 & 20,8 & 276,0 & 10,4 \\
\hline Total & 408 & 100 & 496 & 100,0 & 212,0 & 100,0 & 4944,0 & 100,0 & 1732,0 & 100,0 & 2644,0 & 100,0 \\
\hline
\end{tabular}

$\mathrm{N}=$ número de individuos, $\mathrm{M}=$ mantillo, $\mathrm{P} 1=$ profundidad entre 0 y $10 \mathrm{~cm}, \mathrm{P} 2=$ profundidad ente 10 y $20 \mathrm{~cm}$.

Con base en los resultados anteriores, se observa que los órdenes Hymenoptera, Acari y Coleoptera son los más prevalentes en los sistemas evaluados, con diferencias en Riqueza y AR entre en el bosque y café, lo cual se debe a las diferencia entre los microclimas presentes en cada sistema entre estos sistemas biológicos y al tipo de manejo dado al cultivo de café en cada uno de los municipios (Peredo et al., 2009).

Santos et al. (2018) encontraron una correlación positiva entre el sistema de producción de café y la diversidad de la macrofauna del suelo. 
Obtuvieron 3.354 individuos y entre los taxones más representativos, fueron Oligochaeta, Coleoptera, Gastropoda, Hymenoptera, Isoptera y Chilopoda, independientemente del sistema de cultivo. Las prácticas agrícolas en café tienen un notable cambio en la abundancia de la macrofauna, debido a que el sistema proporciona servicios para la actividad de la macrofauna del suelo (Karungi et al., 2018).

La riqueza de especies está en relación positiva con la complejidad vegetal; la cual determina una mayor cantidad de microhábitats y de recursos disponibles (Rivas y Schoereder, 2007). Suárez et al. (2019) evaluaron la ocurrencia de macrofauna en suelos cafeteros con diferentes sistemas de manejo, en total, 17.109 individuos fueron registrados, siendo el grupo Oligochaeta el más representativo, independientemente del sistema de producción de café.

En cuanto al índice de Shannon Weaver $(\mathrm{H})$, se observaron diferencias significativas entre sistemas en Consacá, La Florida y Sandoná, entre profundidades en La Florida y Sandoná. La ISP solo fue significativa en Sandoná. En el índice se Simpson (Ds) existieron diferencias significativas entre sistemas en Consacá, entre profundidades en Sandoná, sitio donde la ISP fue significativa (Tabla 1).

En Consacá el valor de $\mathrm{H}$ para el bosque fue estadísticamente superior a café, mientras que entre las profundidades no se observaron diferencias. Un comportamiento similar se observó en Ds con un valor superior en el bosque respecto a café y sin diferencias entre profundidades, lo cual confirma lo obtenido con el índice $\mathrm{H}$. En La Florida, el índice $\mathrm{H}$ fue mayor en el bosque que en café. $M$ y P1 no fueron diferentes y superaron a P2. No se observaron diferencias entre los sistemas y profundidades en Ds. En La Unión no se detectó ningún tipo de diferencia (Tabla 2). En Sandoná, la ISP fue significativa para $\mathrm{H}$ y Ds (Figura 1). Se puede observar que los mayores valores de $\mathrm{H}$ se obtuvieron en $\mathrm{M}$ del sistema bosque, tal como se confirma con el índice Ds, dado que cuando su valor tiende a cero, por lo tanto, la diversidad tiende a ser más alta y si fuera cero, la diversidad tendería hacía el infinito (Kiernan, 2020). Los menores índices de $\mathrm{H}$ se encontraron en bosque y café a P2 (Tabla 1).

Los valores de $\mathrm{H}$ oscilaron entre 0,93 y 1,85 con mayor frecuencia de valores por encima 1,14. El índice $\mathrm{H}$ aumenta a medida que aumenta la Ri, tal como se observa en la Tabla 2, situación que es descrita por Somarriba (1999), quien indica que el índice $\mathrm{H}$ aumenta a medida que aumenta la riqueza y los individuos se distribuyen más homogéneamente entre todas las especies.

Tanto el índice $\mathrm{H}$ como el de Ds han sido utilizados para establecer la diversidad alfa, la cual representa la diversidad de especies a lo largo de un hábitat, y por definición tiene en cuenta tanto la riqueza y la abundancia relativa de especies La gran mayoría de los índices de diversidad-alfa utilizan los valores de riqueza y abundancia relativa, solamente que las operaciones matemáticas de estos valores se organizan de diferentes formas (CarmonaGalindo y Carmona, 2013). En este sentido, los valores de Ds obtenidos a través de los diferentes municipios oscilaron entre 0,23 y 0,53 , con un alto grado de diversidad en los valores de Ds (Tabla 2).

Delgado et al. (2011) publicaron índices de diversidad de 0,39 en un sistema de bosque y de 0,32 en un sistema de café más Inga edulis con mayor AR del orden Coleoptera, a diferencia del sistema monocultivo de café que exhibió valores más bajos de diversidad $(0,22)$, con mayor frecuencia del orden Coleoptera con la familia Scarabaeidae y el orden Oligochaeta con la familia Lumbricidae. Pashanasi (2001) sostiene que en bosques y sistemas agroforestales se presenta mayor diversidad de recursos debido a que los ecosistemas brindan 
sombra y protección del suelo, alta humedad edáfica, bajas temperaturas, aporte de hojarasca y detritos, cuyos elementos contribuyen a la subsistencia de la edafofauna principalmente en los bosques secundarios de regeneración natural.
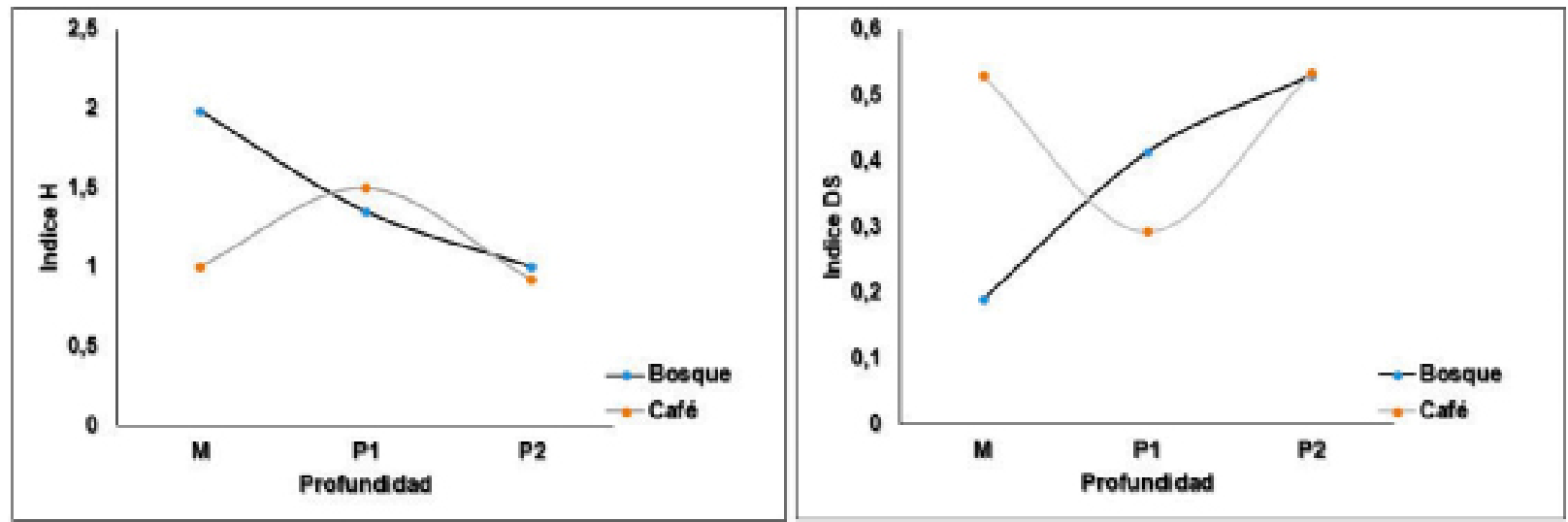

Figura 1. Interacción sistema x profundidad de los índices de Shannon-Weaver $(\mathrm{H})$ y de Simpson (Ds) en el municipio de Sandoná.

\section{CONCLUSIONES}

El sistema bosque presentó mayor diversidad que el sistema café. A excepción de los índices de Shannon-Weaver y Simpson en Sandoná, la interacción sistemas y profundidades fue no significativa. Los órdenes Hymenoptera, Acari y Coleoptera son los más prevalentes en los municipios de Consacá, La Florida, La Unión y Sandoná con diferencias en riqueza y abundancia relativa entre el bosque y café. El comportamiento de los índices ShannonWeaver $(\mathrm{H})$ y de Simpson (Ds) es afectado por la riqueza y la abundancia relativa de los diferentes hábitats evaluados.

\section{Conflicto de Intereses}

Los autores declaran que es un trabajo original y no existió conflicto de intereses de ningún tipo en la elaboración y publicación del manuscrito.

\section{REFERENCIAS}

Aquino, R., Tuesta, C. y Rengifo, E. 2012. Diversidad de mamíferos y sus preferencias por los tipos de hábitats en la cuenca del río Alto Itaya, Amazonía peruana. Rev. Peru. Biol. 19(1): 035-042.

Anderson, J.M. and Ingram, J.S. 1994. Tropical soil biology and fertility: a handbook of methods. CAB International, Wallinford, UK. 238p.

Doi: $\underline{10.2307 / 2261129}$

Amazonas, N.T., Viani, R.A., Rego, M.G., Camargo, F.F. Fujihara, R.T. and Valsechi, O.A. 2018. Soil macrofauna density and diversity across a chronosequence of tropical forest restoration in Southeastern Brazil. Braz. J. Biol. 78(3): 449-456. Doi: 10.1590/1519-6984.169014

Brussaard, L. 2012. Ecosystem services provided by the soil biota. pp. 45-58. En: Wall, D. y Bardgett, R. (Eds.). Soil Ecology and Ecosystem Services. Oxford University Press. Reino Unido. 406 p. 
Cabrera-Mireles, H., Murillo-Cuevas, H.F., Adame-García, J. y Fernández-Viveros, J.A. 2019. Impacto del uso del suelo sobre la meso y macrofauna edáfica en caña de azúcar y pasto. Tropical and Subtropical Agroecosystems. 22: 33-43. https://www.researchgate.net/ publication/333117700

Carmona-Galindo, V.D. y Carmona, T.V. 2013. La diversidad de los análisis de diversidad. Bioma. 14: 20-28.

https://www.researchgate.net/ publication/260192894

Castellanos, L., González, A.F. y Capacho, A.E. 2019. Influencia de los sistemas agroforestales del proyecto Plantar sobre la macrofauna del suelo. Revista Bistua Facultad de Ciencias Básicas. 17(3):105116.

Delgado, G., Burbano, A. y Silva, A. 2011. Evaluación de la macrofauna del suelo asociada a diferentes sistemas con café Coffea arabica L. Revista de Ciencias Agrícolas. 28(1): 91-106. Federación Nacional de Cafeteros de Colombia, Cenicafé - Agroclimatología División de Desarrollo Social, Santa Fe de Bogotá. $138 \mathrm{p}$.

https://biblioteca.cenicafe.org/ bitstream/10778/818/1/lib13731.pdf

García, Y., Ramírez, W. y Sánchez, S. 2014. Efecto de diferentes usos de la tierra en la composición y la abundancia de la macrofauna edáfica, en la provincia de Matanzas. Pastos y Forrajes. 37(3): 313321.

García, A. y Bello, A. 2004. Diversidad de los organismos del suelo y transformaciones de la materia orgánica. I Conferencia Internacional eco-biología del suelo y el compost. SoilACE, León. pp211-212. https://www.uv.mx/personal/tcarmona/ files/2010/08/Garcia-y-Bello-2004.pdf

Gómez, L., Caballero, A. y Baldión, J.V. 1991. Ecotopos cafeteros de Colombia. Federación Nacional de Cafeteros de Colombia, Cenicafé - Agroclimatología División de Desarrollo Social, Santa Fe de Bogotá. 138p.

https://biblioteca.cenicafe.org/ bitstream/10778/818/1/lib13731.pdf
Gizzi, A.H., Álvarez, H.A., Manetti, P.L., López, A.N., Clemente, N.L. y Studdert, G.A. 2009. Caracterización de la meso y macrofauna edáfica en sistemas de cultivo del sudeste bonaerense. CI. Suelo (Argentina). 27(1): 1-9.

https://www.suelos.org.ar/publicaciones/ vol 27n1/Gizzi\%20et\%20al.pdf

Jost, L. 2006. Entropy and diversity. Oikos. 113: 363-375.

Karungi, J., Cherukut, S., ljala, A. R., Tumuhairwe, J. B., Bonabana-Wabbi, J., Nuppenau, E. A and Otte, A. 2018. Elevation and cropping system as drivers of microclimate and abundance of soil macrofauna in coffee farmlands in mountainous ecologies. Applied Soil Ecology.

Doi:10.1016/j.apsoil.2018.08.003

Lavelle, P. 2009. Ecology and the challenge of a multifunctional use of soil. Pesq. agropec. bras., Brasília. 44(8): 803-810. 10.1590/S0100-204X2009000800003

Masin, C.E., Cruz, M.S., Rodríguez, A.R., Demonte, M.J., Vuizot, L.A., Maitre, M.I., Godoy, J.L. y Almada, M.S. 2017. Macrofauna edáfica asociada a diferentes ambientes de un vivero forestal (Santa Fe, Argentina). Ci. Suelo (Argentina) 35(1): 21-33.

https://www.researchgate.net/ publication/320504719

Pashanasi, B. 2001. Estudio cuantitativo de la macrofauna del suelo en diferentes sistemas de uso de la tierra en la Amazonia Peruana. Folia Amazónica. 12:75-97.

Pardo-Locarno, L.C. macroinvertebrados edáficos en agroecosistemas del municipio de Cerrito (Valle), con énfasis en la comunidad de escarabajos Melolonthidae. Tesis Doctoral. Universidad del Valle, Facultad de Ciencias Naturales y Exactas, Programa de Biología. Santiago de Cali. 174p. 
Peredo, S.F., Parada, E., Vega, M. and Barrera, C.P. 2009. Edaphic mesofauna community structure in organic and conventional management of cranberry (Vaccinium sp.) plantations: an agroecological approach. Rev. Cienc. Suelo Nutr. / J. Soil. Sci. Plant Nutr. 9(3): 236-244.

Rivas, C. and Schoereder, J. 2007. Ant communities, environmental characteristics and their implications for conservation in the Brazil Pantanal. Biodiversity and Conservation. 16: 15111520.

Santos, J. B. D., Ramos, A. C., Azevedo, R., Oliveira, L. C. I. D., Baretta, D. and Cardoso, E. J. B. N. 2018. Soil macrofauna in organic and conventional coffee plantations in Brazil. Biota Neotropica, 18.

Socarrás, A. 2013. Mesofauna edáfica: indicador biológico de la calidad del suelo. Pastos y Forrajes. 36 (1): 5-13.

Socarrás-Rivero, A.A. 2018. Diversidad de la mesofauna edáfica en tres usos del suelo en la provincia Mayabeque, Cuba. Pastos y Forrajes. 41(2): 123-130.
Somarriba, E. 1999. Diversidad Shannon. Agroforesteria en las Américas. 6(23): 7274.

https://www.researchgate.net/ publication/324164467

Steel, R.G. and Torrie, J.H. 1960. Principles and procedures of statistics. McGrawHil1, New York. 481p.

Suárez, L., Rodríguez Pinto, S, Cuarán, and Salazar, J.C. 2019. Soil Macrofauna and Edaphic Properties in Coffee Production Systems in Southern Colombia. Floresta e Ambiente, 26(3): p1-8.

Doi: 10.1590/2179-8087.033418

Zerbino,M.S.2010. Evaluación delamacrofauna del suelo en rotaciones cultivos-pasturas con laboreo convencional. Acta Zool. Mex. 26(2): 189-202. 